\title{
Isolated eyelid closure myotonia in two families with sodium channel myotonia
}

\author{
B. C. Stunnenberg • H. B. Ginjaar • J. Trip • \\ C. G. Faber • B. G. van Engelen • G. Drost
}

Received: 7 August 2009 / Accepted: 5 October 2009/Published online: 30 October 2009

(C) The Author(s) 2009. This article is published with open access at Springerlink.com

\begin{abstract}
Sodium channelopathies $(\mathrm{NaCh})$, as part of the non-dystrophic myotonic syndromes (NDMs), reflect a heterogeneous group of clinical phenotypes accompanied by a generalized myotonia. Because of recent availability of diagnostic genetic testing in NDM, there is a need for identification of clear clinical genotype-phenotype correlations. This will enable clinicians to distinguish NDMs from myotonic dystrophy, thus allowing them to inform patients promptly about the disease, perform genetic counseling, and orient therapy (Vicart et al. Neurol Sci 26:194-202, 2005). We describe the first distinctive clinical genotypephenotype correlation within $\mathrm{NaCh}$ : a strictly isolated eyelid closure myotonia associated with the L250P mutation in SCN4A. Using clinical assessment and needle EMG, we identified this genotype-phenotype correlation in six L250P patients from one $\mathrm{NaCh}$ family and confirmed this finding in another, unrelated $\mathrm{NaCh}$ family with three L250P patients.
\end{abstract}

Electronic supplementary material The online version of this article (doi:10.1007/s10048-009-0225-x) contains supplementary material, which is available to authorized users.

B. C. Stunnenberg $\cdot$ B. G. van Engelen $\cdot$ G. Drost $(\bowtie)$ Department of Neurology, Neuromuscular Centre Nijmegen, Radboud University Nijmegen Medical Centre,

Reinier Postlaan 4, PO-box 9101, 6500 HB Nijmegen,

The Netherlands

e-mail: G.Drost@neuro.umcn.nl

H. B. Ginjaar

Department of Human and Clinical Genetics,

Leiden University Medical Centre,

Leiden, The Netherlands

J. Trip · C. G. Faber

Department of Neurology, Maastricht University Medical Centre,

Maastricht, The Netherlands
Keywords Non-dystrophic myotonic syndromes .

Sodium channelopathies $\cdot \mathrm{SCN} 4 \mathrm{~A} \cdot \mathrm{Na}_{\mathrm{v}} 1.4$.

Genotype-phenotype correlation

\section{Introduction}

Sodium channelopathies $(\mathrm{NaCh})$, as part of the nondystrophic myotonic syndromes (NDMs), give rise to a spectrum of clinical heterogeneous phenotypes corresponding with numerous causative mutations. About 50 different sodium channel mutations have so far been identified in the SCN4A gene encoding the skeletal muscle sodium channel. In contrast to myotonic dystrophy (MD) that is characterized by progressive muscle wasting and systemic manifestations, complaints in NDMs are particularly caused by myotonia [2] The clinical phenotypes of sodium $(\mathrm{NaCh})$ and chloride $(\mathrm{ClCh})$ channelopathies have recently been redefined: a higher frequency and severity of myotonia in the eyelid muscles in $\mathrm{NaCh}$, together with the presence of transient paresis (in $\mathrm{ClCh}$ only) and paradoxical myotonia (in $\mathrm{NaCh}$ only) have been reported [3]. However, clinical differentiation between $\mathrm{NaCh}$ and $\mathrm{ClCh}$ remains complicated because these symptoms are always accompanied by a certain degree of generalized myotonia. Moreover, identification of genotype-phenotype correlations at the level of individual mutations is limited.

\section{Case report}

The index patient, a 60-year-old woman (see Fig. 1a: patient II-2, family 1), was referred to our clinic with complaints primarily consisting of cramp-like sensations in the muscles of the eyelids, for she could not open her eyes 
a

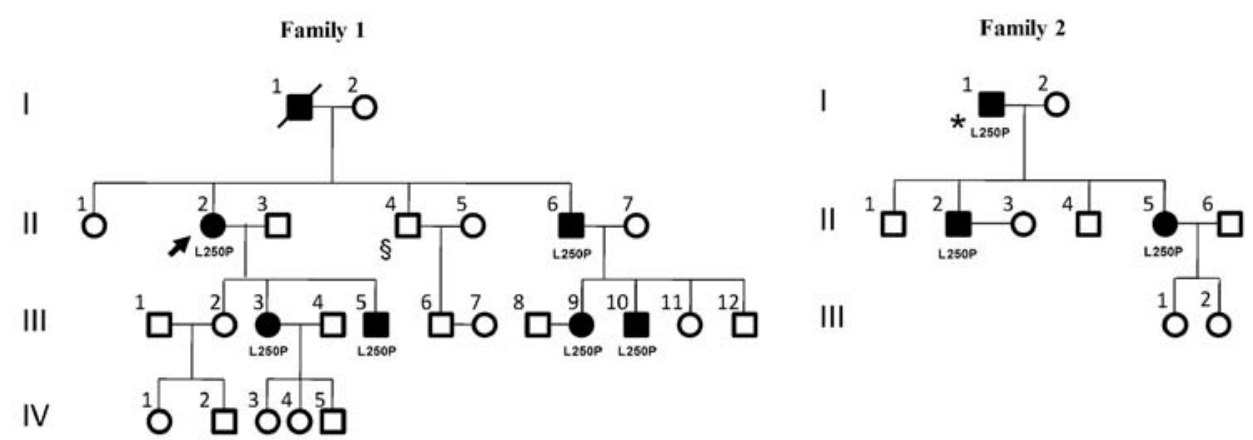

b

C
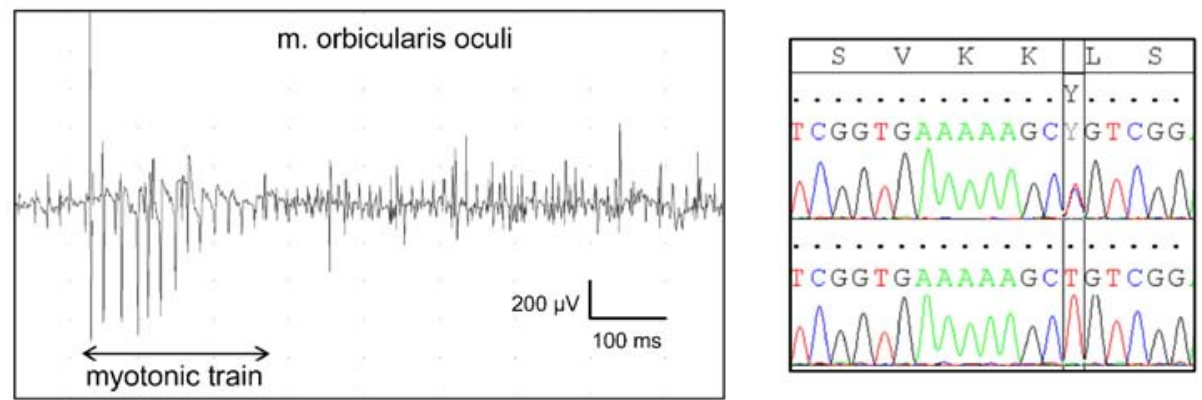

Fig. 1 a Pedigrees of the two Dutch families carrying the L250P mutation and segregation of the mutation within the pedigrees. Family 1 is the family of the index patient described in this report. In Family 2 the L250P mutation was previously reported as a causative mutation for sodium channel myotonia (SCM) associated with a warm-up phenomenon. However, no further detailed phenotypic characteristics were reported in these patients [2]. Pedigree symbols: circles female, squares male, slashed square deceased, closed symbols affected individuals, arrow the index patient, section sign refused participation in this study, asterisk see supplemental data: Videos 1 and 2 showing action myotonia in this patient. b Needle EMG segment shows an isolated myotonic train with changes in frequency and amplitude;

normally after strong eyelid closure, especially after crying, sneezing, and after exposure to cold, for example cold wind. These symptoms had first developed in early childhood and did not improve as she grew older. She had never experienced episodes of weakness.

Standardized neuromuscular examination revealed the presence of action myotonia in the eyelid closure muscles: after forceful contraction of the $\mathrm{m}$. orbicularis oculi had been maintained for several seconds the patient was unable to open her eyelids and compensatory frontal muscle contraction was needed to open the eyes. This delay passed off gradually after warming up with repetitive contractions. Clinical signs indicating myotonic involvement of the $\mathrm{m}$. levator palpebrae and the superior tarsel muscle (the eyelid opening muscles) were not present: (1) lid lag sign, a limitation of upper eyelid descent on downgaze, (2) retraction sign, upper eyelid retraction above the corneoscleral limbus while looking straight ahead. Ocular movements were normal and no facial weakness was found. No clinical signs of nonocular myotonia, such as grip action recorded from the $\mathrm{m}$. orbicularis oculi in the index patient (II-2, family 1) after myotonic activity was triggered by needle movement. The myotonic train is preceded and followed by motor unit activity of the m. orbicularis oculi. c Results of mutational analysis. The upper sequence within the electropherogram represents the sequence of the index patient (II-2, family 1) encompassing the c.749T $>\mathrm{C}$ mutation (p.L250P). The mutation is indicated with an arrow. The lower sequence represents the control sequence. The L250P mutation was confirmed by direct nucleotide sequence analysis of the $S C N 4 A$ gene in the affected individuals that are marked as carriers of the L250P mutation in Fig. 1a

mytonia in the hand flexor muscles and percussion myotonia of the thenar and extensor forearm muscles, were observed. There was no muscle weakness or wasting. General investigations including serum sodium, potassium, and creatine kinase were normal.

Needle electromyography (EMG) showed intense myotonic discharges in the $\mathrm{m}$. orbicularis oculi (Fig. 1b), in sharp contrast to the other muscles investigated (left $\mathrm{m}$. biceps brachii, right $\mathrm{m}$. interosseus dorsalis, right $\mathrm{m}$. rectus femoris, and the left $\mathrm{m}$. tibialis anterior), which showed minimal or no myotonic discharges. Mutation screening for myotonic dystrophy types 1 and 2 were negative. Screening of the sodium channel SCN4A gene identified the L250P sodium channel mutation (Fig. 1c).

\section{Methods and results}

Five relatives of the index patient who reported no complaints of generalized or eyelid myotonia and five 
relatives who reported eyelid closure myotonic symptoms but no generalized myotonic symptoms (Fig. 1a: family 1) were examined through clinical assessment, needle EMG, and genetic screening. Blood samples had been collected after obtaining written informed consent and ethic approval was provided by the medical ethics committee of the Radboud University Nijmegen Medical Centre. Direct nucleotide sequence analysis confirmed the presence of the L250P mutation only in the five affected relatives. The asymptomatic relatives had no mutation at this site. Additionally, three L250P patients from a previously reported L250P family (Fig. 1a: Family 2) were clinically reevaluated and examined with needle EMG [4]. In all nine L250P patients, eyelid closure myotonia was aggravated by exercise and cold temperatures and the clinical assessment revealed the presence of action myotonia in the $\mathrm{m}$. orbicularis oculi in all nine (see supplemental data: Video 1), in the absence of action myotonia in the hand flexor muscles (Video 2) except for one patient (Fig. 1a: patient III-5 family 1). The needle EMG demonstrated continuous myotonic discharges in the $\mathrm{m}$. orbicularis oculi with minimal or no myotonic discharges in the other muscles examined (left $\mathrm{m}$. biceps brachii, right $\mathrm{m}$. interosseus dorsalis, right $\mathrm{m}$. rectus femoris, and the left $\mathrm{m}$. tibialis anterior) (Table 1).

\section{Discussion}

Until now, only two specific $S C N 4 A$ mutations in $\mathrm{NaCh}$ have been related to a distinctive clinical genotype-phenotype: (1) the M1476I mutation associated with severe painful myotonia and a clear warm-up phenomenon and (2) the N1297K mutation associated with a severe neonatal form of $\mathrm{NaCh}$ with severe desaturation, early-onset muscle hypertrophy, psychomotor retardation, and fatal outcome $[5,6]$.

In contrast to these two clinical phenotypes that are typically characterized by generalized myotonia and distinctive additional features, we report on the first distinctive clinical genotype-phenotype correlation within the sodium channelopathies that we found to be associated with an isolated eyelid closure myotonia due to the L250P mutation. Being at the mild end of the $\mathrm{NaCh}$ spectrum, this phenotype is characterized by intense myotonia of the $\mathrm{m}$. orbicularis oculi with a warm-up phenomenon and minimal or no myotonia elsewhere.

The pathogenic status of this mutation is clear: the L250P mutation in the $S C N 4 A$ gene results in a substitution of the highly conserved leucine residue into a proline residue in the $\alpha$-subunit of the skeletal muscle voltagegated sodium channel $\left(\mathrm{Na}_{\mathrm{v}} 1.4\right)$. In vitro electrophysiological studies using patch-clamp analysis showed that mutations occurring in the voltage sensor domains of the channel cause myotonia through a disruption of fast inactivation.

In vivo, this results in hyperexcitability of the muscle sarcolemmal membrane, causing the subsequent myotonia [1].

However, the question remains why in patients with the L250P mutation myotonia is almost exclusively restricted to the m. orbicularis oculi. Notwithstanding recent studies showing more neuromuscular junctions with clustering of

Table 1 Phenotypic features of two Dutch families carrying the L250P mutation

\begin{tabular}{|c|c|c|c|c|c|c|c|c|c|c|c|c|}
\hline \multicolumn{4}{|c|}{ General } & \multicolumn{6}{|c|}{ Triggering factors of myotonia } & \multicolumn{2}{|l|}{ Action myotonia } & \multirow{2}{*}{$\begin{array}{l}\text { Myotonia } \\
\text { Myotonic } \\
\text { discharges }\end{array}$} \\
\hline No & Sex & Age (years) & SCM onset & $\mathrm{C}$ & E & ES & $\mathrm{CM}$ & $\mathrm{S}$ & $\mathrm{P}$ & Eyelid muscles & $\begin{array}{l}\text { Hand flexor } \\
\text { muscles }\end{array}$ & \\
\hline
\end{tabular}

Family 1

\begin{tabular}{|c|c|c|c|c|c|c|c|c|c|c|c|c|}
\hline II-2 & $\mathrm{F}$ & 60 & Childhood & + & - & - & - & - & + & Present & Absent & m. orbicularis oculi \\
\hline II-6 & M & 56 & Childhood & + & + & + & - & - & - & Present & Absent & m. orbicularis oculi \\
\hline III-3 & $\mathrm{F}$ & 34 & Neonatal & + & + & + & + & + & + & Present & Absent & m. orbicularis oculi \\
\hline III-5 & M & 23 & Childhood & + & + & - & - & - & - & Present & Present $^{\mathrm{a}}$ & m. orbicularis oculi \\
\hline III-9 & $\mathrm{F}$ & 23 & Neonatal & + & - & - & - & - & - & Present & Absent & m. orbicularis oculi \\
\hline III-10 & M & 19 & Neonatal & + & + & - & - & - & - & Present & Absent & m. orbicularis oculi \\
\hline \multicolumn{13}{|c|}{ Family 2} \\
\hline I-1 & M & 73 & Childhood & + & + & - & - & + & - & Present & Absent & m. orbicularis oculi \\
\hline II-2 & M & 48 & Childhood & + & + & - & - & - & - & Present & Absent & m. orbicularis oculi \\
\hline II-5 & $\mathrm{F}$ & 41 & Childhood & + & + & - & - & + & + & Present & Absent & m. orbicularis oculi \\
\hline
\end{tabular}

$F$ female, $M$ male, $C$ cold, $E$ exercise, $E S$ (emotional) stress, $C M$ carbohydrate-rich meal, $S$ sleep deprivation, $P$ pregnancy

${ }^{a}$ In patient III-5 (family 1) minor action myotonia was clinically observed in the hand flexor muscles without the presence of actual symptoms related to hand or other nonocular myotonia 
the voltage-gated sodium channels at the sarcolemmal membrane in human facial muscles, additional modifying genes must play a large role in this resultant phenotype [7, 8].

In view of our case report, clinicians should be aware that patients with sodium channelopathies may present with an isolated eyelid closure myotonia. In these $\mathrm{NaCh}$ cases we recommend $S C N 4 A$ screening with particular attention for the L250P mutation. Besides, testing for eyelid closure myotonia is a very important clinical test to distinguish between MD and NDMs because, unlike NDMs, MD is characterized by a severe degree of weakness of the eyelid opening muscles and not by eyelid closure myotonia [9].

Acknowledgment The authors are grateful to all family members that participated in this study and would like to thank D.J. Verbove, A. Verwer, and H. Janssen for their expert technical assistance.

Disclosure The authors report no conflict of interest.

Open Access This article is distributed under the terms of the Creative Commons Attribution Noncommercial License which permits any noncommercial use, distribution, and reproduction in any medium, provided the original author(s) and source are credited.

\section{References}

1. Vicart S, Sternberg D, Fontaine B et al (2005) Human skeletal muscle sodium channelopathies. Neurol Sci 26:194-202

2. Mankodi A, Thornton CA (2002) Myotonic syndromes. Curr Opin Neurol 15:545-552

3. Trip J, Drost G, Ginjaar I et al (2009) Redefining the clinical phenotypes of non-dystrophic myotonic syndromes. J Neurol Neurosurg Psychiatry 80:647-652

4. Trip J, Drost G, Verbove DJ et al (2008) In tandem analysis of CLCN1 and SCN4A greatly enhances mutation detection in families with non-dystrophic myotonia. Eur J Hum Genet 16:921-929

5. Rossignol E, Mathieu J, Thiffault I et al (2007) A novel founder SCN4A mutation causes painful cold-induced myotonia in FrenchCanadians. Neurology 69:1937-1941

6. Gay S, Dupuis D, Faivre L et al (2008) Severe neonatal nondystrophic myotonia secondary to a novel mutation of the voltagegated sodium channel (SCN4A) gene. Am J Med Genet A $146: 380-383$

7. Caldwell JH (2000) Clustering of sodium channels at the neuromuscular junction. Microsc Res Tech 49:84-89

8. Happak W, Liu J, Burggasser G et al (1997) Human facial muscles: dimensions, motor endplate distribution, and presence of muscle fibers with multiple motor endplates. Anat Rec 249:276-284

9. Harper P, van Engelen B, Eymard B, Wilcox DE (2004) Myotonic dystrophy: present management, future therapy. Oxford University Press, New York 